\title{
A Niosomal Gel of Cefoperazone Sodium for Topical Application
}

\author{
NAVEEN Kumar $\mathrm{R}^{1}$, SAYANI Bhattacharyya ${ }^{*}$ \\ 1Krupanidhi College of Pharmacy, Department of Pharmaceutics, Bangalore, India
}

\begin{abstract}
The present study endeavors to prepare a niosomal gel of Cefoperazone sodium (CFS), as a novel dermal delivery for the treatment of skin infections. CFS loaded niosomes were prepared using different molar ratio of Tween 80 and Cholesterol by ether injection method using experimental design. The optimized formula was evaluated for DSC, XRPD and AFM. A niosomal gel with the optimized formulation was prepared in Carbopol 934 and were evaluated for gelling properties, in-vitro release, ex-vivo permeation and skin irritation study on rats. Quality by design was successfully executed to get stable (Zeta potential $-30 \mathrm{mV})$, nano sized $(365.3 \mathrm{~nm}$ ) niosomal vesicles. The niosomal gel of CFS showed a $\mathrm{pH}$ around 5.5 , and a viscosity of $84.13 \pm 0.25 \mathrm{cps}$, enhanced permeation and no skin irritation. Hence, the study depicts that a superior site-specific delivery of CFS can be achieved with a niosomal gel of the drug in the treatment of skin infections.
\end{abstract}

Keywords: Cefoperazone sodium, custom design, niosomes, niosomal gel, ex-vivo evaluation

\section{INTRODUCTION}

Niosomes are hydrated non-ionic vesicles of surfactant having the unique potential to entrap both hydrophilic and lipophilic drugs. They are unilamellar or multilamellar vesicles of surfactants with cholesterol or its derivatives, enclosed by an aqueous compartment ${ }^{1}$. The self-assembling properties of surfactants on hydration are responsible for formation of shapes like micelles or planar lamellar bilayer of microscopic and nanoscopic vesicles ${ }^{2}$. They are osmotically stable, non-immunogenic, biocompatible, biodegradable, and act as permeation en-

${ }^{*}$ Correspondence Author:

E-mail: sayanibh@gmail.com,

Phone: 9845561865

ORCIDs:

Naveenkumar R: 0000-0002-6629-7009

Sayani Bhattacharyya: 0000-0002-4013-4316

(Received 23 July 2020, accepted 23 February 2021) 
hancers ${ }^{3}$. They can easily adsorb or fuse with stratum corneum and can pass through the intra epidermal channels, and diffuse deep into the skin to produce systemic effects. They can increase the fluidity of the skin membrane and results in enhancement in the permeability of drugs when applied topically ${ }^{4}$.

Cefoperazone sodium, a semi synthetic broad-spectrum antibacterial drug used in the infections of skin caused by Pseudomonas aeruginosa, Streptococcus pyogenes and Staphylococcus aureus ${ }^{5}$. It is administered in divided doses of 2 to $4 \mathrm{gm}$ intravenously per day depending on the severity. The drug has low serum half-life (2hours) and bound mostly in the plasma protein which is also reported to be dose dependent ${ }^{6}$. Therefore, an approach to develop a topical formulation of cefoperazone sodium could be beneficial in the treatment of skin infections considering localization of the drug at the site of action with improved patient compliance. As per the reported data, the drug has a low $\log \mathrm{P}$ value ${ }^{6}$, therefore, encapsulation of the drug in the niosomal vesicle could improve permeation of the drug through the skin. The drug can be encapsulated in the aqueous core of the nonionic vesicles. Cholesterol which is a common component in biological membrane, can influence the permeability and fusion of the vesicles through the stratum corneum, can be added to improvise the bilayer property of the nonionic surfactant vesicle ${ }^{7}$. Several studies have reported that the residence time of drug in stratum corneum was enhanced through niosomal delivery as it altered the horny layer properties ${ }^{8}$ and enhance the stability of the entrapped drug ${ }^{9}$.

Hence, the present study discusses on the development and evaluation of niosomal gel of cefoperazone sodium with targeting of the drug at the site of infection to provide better efficacy and patient compliance over the conventional dosage forms.

\section{METHODOLOGY}

\section{Materials}

Cefoperazone sodium (CFS) was gifted from Aurobindo Pharma Ltd, Hyderabad, India. Cholesterol was obtained from Loba Chemie, Mumbai, India. Carbopol-940 was purchased from SD Fine Chemicals Limited, Mumbai, India. Rest of the used chemicals and reagents were of analytical grade.

\section{Methods}

\section{Employment of custom design in the methods of preparation of niosomes}

The niosomes were prepared by ether injection method. The various process parameters like rate of injection, volume of injection and the property of the 
materials can affect the encapsulation efficiency and the vesicular size of niosomes ${ }^{10}$. Presence of cholesterol plays an important role in bilayer stability of niosome and entrapment of drug ${ }^{1}$. The software JMP version 13 was availed to estimate the effect of surfactant and cholesterol ratio, and process parameters on the response on niosomes vesicle size, and entrapment efficiency with twelve experimental runs through custom design. A two level (low (-1) and high level $(+1)$ ) testing of each variable was done against the responses. From the initial screening study and review of reported literature the composition of surfactant: cholesterol molar ratio (X1) was varied from 6:4 to 8:6, and the process parameters, the rate of injection( $\left.\mathrm{X}_{2}\right)$ and the hydration volume $\left(\mathrm{X}_{3}\right)$ were varied from 0.5 to $1.5 \mathrm{~mL} / \mathrm{min}$, and $10 \mathrm{~mL}$ to $30 \mathrm{~mL}$ respectively.

\section{Ether Injection Method for preparation of cefoperazone loaded niosomes (CFS-NIO)}

A solution with appropriate molar ratio of cholesterol and surfactant (Tween $80)$ in ether was prepared. An aqueous solution of the drug (0.067\%w/v) was prepared in phosphate citrate buffer at $\mathrm{pH}$ 3. The organic phase was slowly injected into the preheated aqueous solution of the drug maintained at $60{ }^{\circ} \mathrm{C}$ through a syringe pump. The vaporization of ether leads to the spontaneous formation of lamellar vesicles of the surfactants containing a drug ${ }^{11}$. The resultant drug-loaded niosome formulations (CFS-NIO) were equilibrated at room temperature and stored overnight at $4^{\circ} \mathrm{C}$ in the refrigerator prior taken for further evaluation.

\section{Evaluation of niosomes}

\section{Particle size distribution}

Horiba SZ-10o nanoparticle size analyzer was used to determine the particle size of the niosomes. After suitable dilution with double distilled water, the sample of niosomal dispersion was placed in disposable cuvettes for particle size measurements at a scattering angle of $90^{\circ}$ at $25.2^{\circ} \mathrm{C}^{12}$. Three trials were done for determining the average particle size of each formulation.

\section{Estimation of zeta potential}

The zeta potential of all the CFS-NIOs was measured in Horiba SZ10o. After dilution of the samples with doubled distilled water, three measurements were carried out for each sample at $25 \cdot 2^{\circ} \mathrm{C}^{12}$.

\section{Estimation of drug entrapment efficiency}

Entrapment efficiency of all the CFS-NIO formulations were determined by centrifugation method. Niosomal formulations were centrifugated at $14000 \mathrm{rpm}$ 
for $40 \mathrm{~min}$ at $4^{\circ} \mathrm{C}$. The supernatant layer was separated to estimate the unentrapped drug $\left(\mathrm{F}_{\mathrm{drug}}\right)$. A $0.22 \mu \mathrm{m}$ AST syringe filter was used to filter the supernatant layer. It was suitably diluted with phosphate buffer $\mathrm{pH} 5.5$ and analyzed by UV-visible spectroscopy at $\lambda \max 286 \mathrm{~nm}$ to estimate the free drug. The analytical method for assay was validated prior by establishing a linearity range between $5-25 \mu \mathrm{g} / \mathrm{mL}$ with a regression coefficient value of $\left(\mathrm{R}^{2}\right) 0.9991$. The method was found to be accurate, precise, and robust with relative standard deviation (RSD) of less than $2 \%$. The total drug $\left(\mathrm{T}_{\text {drug }}\right.$ ) was estimated by lysing the equal volume of niosomal dispersion in methanol followed by centrifugation and analyzed spectrophotometrically at 286nm. A blank niosomal dispersion of each formulation was treated in the similar way and used as blank to nullify the effect of excipients in absorbance.

The entrapment efficiency was determined using the following formula ${ }^{8,13}$. Each result represents an average of three trials with standard deviation.

$\%$ Drug entrapment efficiency $=($ Tdrug - Fdrug $) .100 /$ Tdrug

\section{Atomic force microscopy (AFM)}

The surface morphology of optimized CFS-NIO was analyzed by atomic force microscopy (AFM). The sample was diluted with deionized water to make a nano dispersion. A drop of this dispersion was placed over a glass slide and covered with a coverslip. The sample was air dried to remove moisture from the sample. AFM was carried out by using Park systems NX-10 AFM at $80 \mathrm{kV}$. The images were recorded in $2 \mathrm{D}$ and $3 \mathrm{D}$ scales ${ }^{14}$.

\section{Freeze drying of optimized niosomes formulation}

Lyophilization was done for the optimized drug loaded niosomal suspension. The lyoprotectants mannitol $(15 \% \mathrm{w} / \mathrm{w})$ and dextrose $(5 \% \mathrm{w} / \mathrm{w})$ were added to the niosomal suspension to yield a sugar: lipid ratio of 3:1. The suspension was subjected to freezing at -80'C for $24 \mathrm{~h}$ in a deep freezer (REMI ULT-90) followed by freeze drying in (LABCONCO freeze-dryer, Free-Zone 4.5, USA) at a preset condition of vacuum pressure maintained at a $54 \times 10^{-3}$ bar and surface temperature held at $-54^{\prime} \mathrm{C}$ for $8 \mathrm{~h}^{15}$. The freeze-dried samples were collected and stored in a tightly closed glass vials in a desiccator. The lyophilized sample (LYP-CFS-NIO) were further taken for thermal analysis, x-ray powder diffraction, and surface morphology study. 


\section{Differential scanning colorimetry (DSC)}

DSC was used for thermal analysis of pure drug (CFS) and LYP-CFS-NIO formulation. The instrument used was DSC-60, Shimadzu, Japan. Each sample was placed in an aluminum pan, sealed with pierced lids, and heated at $5{ }^{\circ} \mathrm{C} / \mathrm{min}$ over a temperature range of $30-300{ }^{\circ} \mathrm{C}$ under a nitrogen purging of $40 \mathrm{~mL} / \mathrm{min}^{16}$.

\section{$X$-ray powder diffraction (XRPD)}

XRPD patterns of the pure drug (CFS) and LYP-CFS-NIO formulation were obtained using an X-ray diffractometer (X' Pert3 powder-Malvern Panalytical). A $\mathrm{Cu}$-Ka radiation was used to analyze the sample between $4^{\circ}$ and $100^{\circ} 2 \theta$ with a scan rate of $4^{\circ} / \mathrm{min}$. Voltage and current were maintained at $40 \mathrm{kV}$ and $30 \mathrm{~mA}$, respectively ${ }^{16}$.

\section{Preparation of niosomal gel CFS-NIO-gel}

The LYP-CFS-NIO equivalent to $0.01 \% \mathrm{w} / \mathrm{w}$ drug was incorporated into a gel base of Carbopol 934(2\%w/w). The required quantity of Carbopol 934 in a small amount was dispersed in distilled water and hydrated for $4 \mathrm{~h}$. Propylene glycol $(7 \% \mathrm{w} / \mathrm{w})$ was added to the hydrated base. Triethanolamine solution $(1 \% \mathrm{w} / \mathrm{v})$ was used to adjust the $\mathrm{pH}$ of the base to 5.5. Finally, distilled water was added to adjust the gel weight to $\log ^{17}$. A gel containing pure drug of the equivalent quantity was prepared in the same manner for comparative evaluation.

\section{Evaluation of gel}

\section{pH of gel}

Digital $\mathrm{pH}$ meter (Digisun Electronics System) was used to determine $\mathrm{pH}$ of CFS-NIO-gel. It was calibrated before its use. The $\mathrm{pH}$ measurement was made in triplicates ${ }^{18}$.

\section{Viscosity of gel}

The viscosity of CFS-NIO-gel was determined at $25^{\circ} \mathrm{C}$ by using brook field viscometer. The niosomal gel $(20 \mathrm{~g})$ was rotated at $10 \mathrm{rpm}$ with spindle 2 . Three trials were made for the estimation of viscosity ${ }^{18}$.

\section{Drug content for drug-loaded niosomal gel}

A known quantity of the CFS-NIO-gel was taken in the Eppendorf tube and diluted with methanol and kept for vortex mixing for 1ominutes. An aliquot was withdrawn, filtered with syringe filter, diluted suitably with phosphate buffer $\mathrm{pH} 5.5$, and estimated for drug content spectrophotometrically at $286 \mathrm{~nm}^{19,20}$. Three trials were run to confirm the estimation. 


\section{Spreadability}

Spreadability was determined based on slide and drag method. An excess of the CFS-NIO-gel was placed on a glass slide. Another slide was placed over it. To spread the gel uniformly on slide, a weight of $500 \mathrm{~g}$ was placed on the top of the slides for few minutes. Spreadability was determined by measuring the time to drag a fixed distance after placing a weight of $100 \mathrm{~g}$ on the slides ${ }^{21,22}$. Spreadability was calculated by

$$
\text { Spreadability }=\frac{\text { Mass on the slide } \mathrm{X} \text { length of slide }}{\text { Time taken for complete separation of slides }} .
$$

\section{In-vitro drug release and kinetic study}

The in-vitro drug release of niosomal gel was carried out in Franz diffusion cell apparatus using $0.22 \mu \mathrm{m}$ dialysis membrane from Himedia. The dialysis membrane was soaked in phosphate buffer $\mathrm{pH} 7.4$ overnight prior use. The receptor compartment was filled with $45 \mathrm{~mL}$ of phosphate buffer of $\mathrm{pH}$ 7.4. A quantity of $1 \mathrm{~g}$ gel was placed in the donor compartment. The whole assembly was kept over magnetic stirring and the temperature of the assembly was maintained at $37^{\circ} \mathrm{O} .5^{\circ} \mathrm{C}$. An aliquot of $1 \mathrm{~mL}$ was withdrawn at a suitable time interval and replenished with equal volume of fresh media to maintain sink conditions. The study was carried out for $8 \mathrm{~h}$. The aliquots after suitable dilution were analyzed spectrophotometrically at $286 \mathrm{~nm}$. The \% cumulative drug release was calculat$\mathrm{ed}^{23}$. The release kinetics data were analyzed for zero order, first order, Higuchi, Korsmeyer-Peppas model through linear regression analysis

\section{Ex-vivo diffusion study}

The ex-vivo studies were executed using the abdominal skin of albino Wistar rats weighing between 250-300gms. To conduct the study an approval from the institutional ethical committee vides the approval number KCP/IAEC/ PCEU/39/2019 was procured in advance. The rats were euthanized using excess of carbon dioxide, the abdominal skin was depilated, and rinsed thoroughly with phosphate buffer of $\mathrm{pH}$ 7.4. A section of the skin was cut and tied to donor compartment of the Franz diffusion cell such that the dorsal side of the skin projecting the donor compartment. The receptor compartment was filled with 45mL phosphate buffer of $\mathrm{pH}$ 5.5. The LYP-CFS-NIO-gel and gel of pure drug containing equivalent drug were taken for permeation study. The receptor compartment was under magnetic stirring. The temperature of the assembly was maintained at $32^{\circ} \mathrm{O} \cdot 5^{\circ} \mathrm{C}$. The samples were withdrawn at constant interval of time for $8 \mathrm{~h}$, the same volume of fresh solution was replaced to maintain sink condition. The withdrawn samples were suitably diluted and analyzed spectro- 
photometrically at $286 \mathrm{~nm}^{24}$. From the data the permeation constant (Kp) and the steady state permeation flux (Jss) were determined.

\section{Skin irritation test}

The skin irritation study of LYP-CFS-NIO-gel was performed on six wistar albino rats. The dorsal and ventral side of the rats were shaved to remove the hairs. Marking was done on both sides. Ventral and the dorsal side served as control and test, respectively. Gel was applied once a day for three consecutive days. The skin irritation was recorded by observation for any skin sensitivity reactions like swelling, redness, and skin rash ${ }^{25,26}$.

\section{RESULTS and DISCUSSION}

\section{Optimization of the custom design through evaluation of particle size, zeta potential and \% entrapment efficiency of CFS-NIO}

The custom design constitutes a radical approach to find the possibility of investigating a high number of variables at different levels with minimal experimentation. The use of center points in the design increased the confidence level and helped to minimize the errors on experimentation. The experimental runs of the twelve formulations resulted the responses \%entrapment efficiency (\%EE), and particle size (PS) as shown in table 1.

Table 1: Factors and observed responses for the custom design

\begin{tabular}{|c|c|c|c|c|c|}
\hline $\begin{array}{c}\text { Formulation } \\
\text { code }\end{array}$ & $\begin{array}{c}\text { Surfactant: } \\
\text { cholesterol } \\
\text { (Molar ratio) } \\
\text { (X1) }\end{array}$ & $\begin{array}{c}\text { Rate of } \\
\text { injection } \\
\text { (mL/min) } \\
\text { (X2) }\end{array}$ & $\begin{array}{c}\text { Hydration } \\
\text { volume (mL) } \\
\text { (X3) }\end{array}$ & $\begin{array}{c}\text { Particles } \\
\text { size(nm) } \\
\text { (PS) }\end{array}$ & $\begin{array}{c}\text { Drug } \\
\text { entrapment } \\
\text { efficiency (\%) } \\
\text { (EE) }\end{array}$ \\
\hline F1 & 1 & 1 & 1 & $317.4 \pm 5.09$ & $98.93 \pm 0.001$ \\
\hline F2 & -1 & -1 & 1 & $416.5 \pm 4.04$ & $98.93 \pm 0.003$ \\
\hline F3 & 0 & 0 & 0 & $499 \pm 0.05$ & $98.89 \pm 0.009$ \\
\hline F4 & 1 & -1 & -1 & $339.8 \pm 3.08$ & $98.56 \pm 0.052$ \\
\hline F5 & -1 & 1 & -1 & $433.5 \pm 6.08$ & $98.76 \pm 0.001$ \\
\hline F6 & -1 & 1 & 1 & $453.9 \pm 7.03$ & $99.11 \pm 0.025$ \\
\hline F7 & 1 & -1 & -1 & $361.3 \pm 9.05$ & $98.41 \pm 0.01$ \\
\hline F8 & 1 & -1 & 1 & $644.3 \pm 507$ & $98.81 \pm 0.085$ \\
\hline F9 & 0 & 0 & 0 & $412.1 \pm 9.44$ & $98.92 \pm 0.096$ \\
\hline F10 & 1 & 1 & -1 & $290.4 \pm 8.06$ & $98.98 \pm 0.034$ \\
\hline F11 & 1 & 1 & 1 & $249 \pm 6.01$ & $98.99 \pm 0.047$ \\
\hline F12 & -1 & -1 & $378.5 \pm 8.08$ & $98.94 \pm 0.011$ \\
\hline
\end{tabular}


Regression analysis was done to generate a relationship between factors and responses using JMP V13 software. The response surface diagrams depicted the significant effect of composition, hydration volume and rate of injection on entrapment efficiency. The particle size of the niosomes were greatly affected with rate of injection and hydration volume. The three-dimensional (3D) response surface graphs represented the most statistically significant variables on the evaluated responses as shown in figure 1.
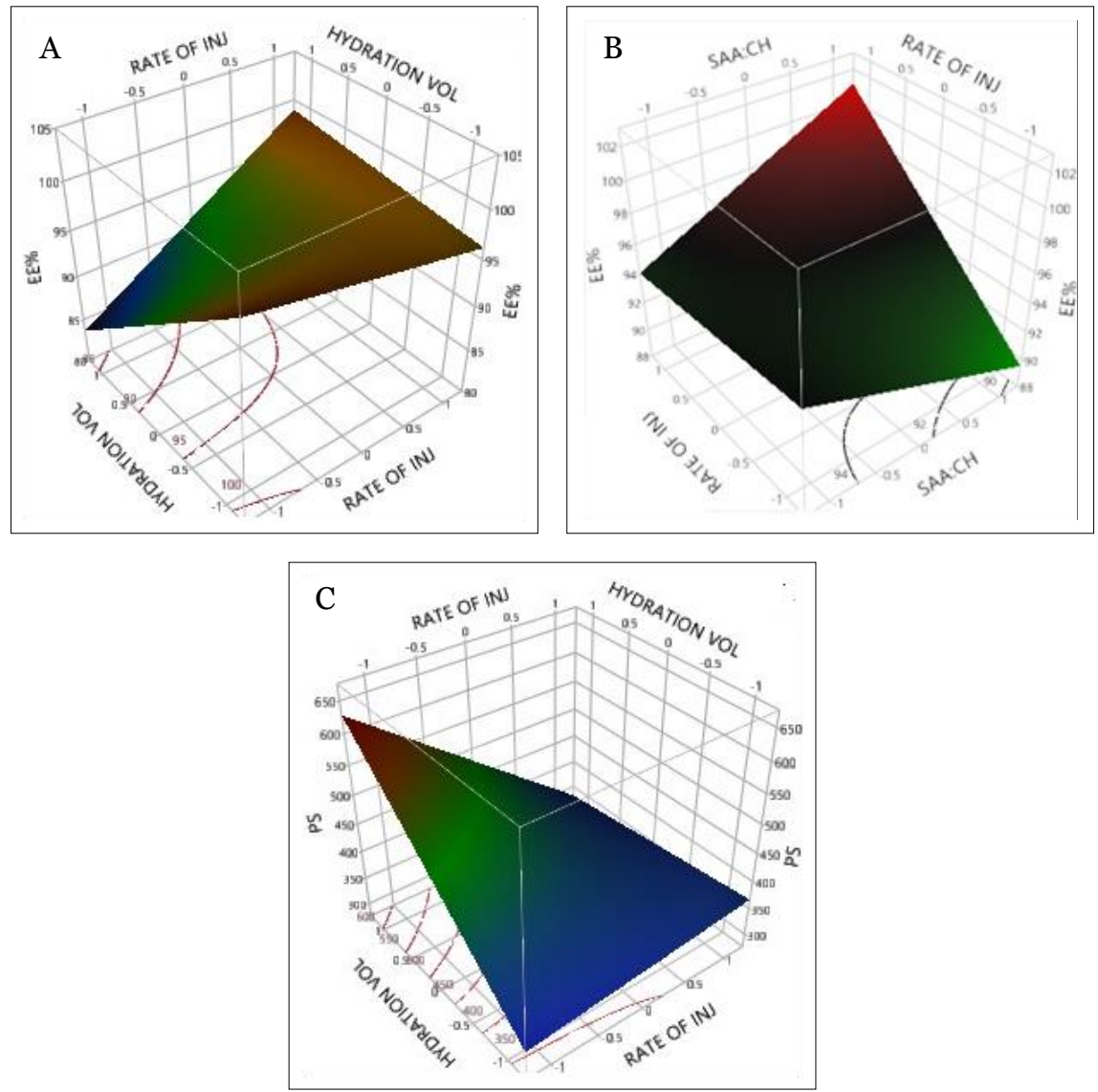

Figure 1: Response surface diagram of variables on the three responses A: \%EE, B: \%EE C:PS

The response surface diagrams revealed that higher the hydration volume the higher the EE. The particle size was significantly affected by the rate of injection. The effect of the various factors on the responses were estimated through parameter sensitivity analysis and listed in table 2. 
Table 2: Parameter sensitivity analysis

\begin{tabular}{|c|c|c|}
\hline Factors & \multicolumn{2}{|c|}{ Prob> t } \\
\hline \multicolumn{2}{|c|}{$\%$ Entrapment Efficiency (EE) } & Particle Size (PS) \\
\hline SAA:CH & $0.042^{\star}$ & 0.088 \\
\hline Rate of injection & $0.036^{*}$ & $0.013^{*}$ \\
\hline Hydration & $0.010^{\star}$ & $0.011^{*}$ \\
\hline
\end{tabular}

*indicates significance

The best-fitted model for the design was found to be the quadratic model when the validation was carried out at a significance level of $\mathrm{P}<0.05$. The ANOVA study showed the model was significant for estimation of the effects of compositions and process parameters on entrapment efficiency and particle size as shown in figure 2 in the predicted vs observed graph. The regression coefficient value of more than 0.9 proved further the significance of the model with respect to all the responses.
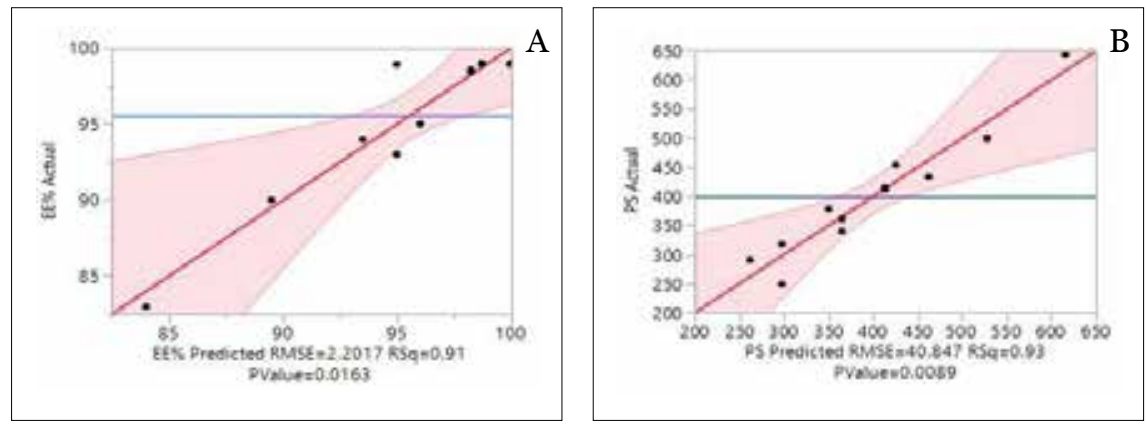

Figure 2: Actual vs predicted plot of variables on the two responses A: \%EE, B: PS.

The model optimization was carried out at desirability of 0.78 and an optimized condition was predicted at a SAA/CH molar ratio of 7:5, rate of injection $1 \mathrm{~mL} /$ min and a hydration volume of $20 \mathrm{~mL}$ to yield a drug loaded niosome with high EE and small PS. The optimized formulation was prepared with the predicted parameters and evaluated for the responses. The listed experimental values of the optimized product prepared are shown in table 3 . The responses of the optimized product were close to the predicted values with low percentage bias, suggesting the rationality and reliability of the model. 
Table 3: Comparison of the predicted and experimental values of the optimal condition

\begin{tabular}{|c|c|c|c|}
\hline Responses & Predicted & Experimental & \%Bias \\
\hline \%Entrapment efficiency & 96.65 & $94 \pm 0.66$ & 2 \\
\hline Particle size $(\mathrm{nm})$ & 374 & $365.3 \pm 0.82$ & 2.94 \\
\hline
\end{tabular}

The zeta potential of the optimized formulations was found to be $-30 \mathrm{mV}$. The particle size and the zeta potential of the optimized formulations are shown in figure 3 .

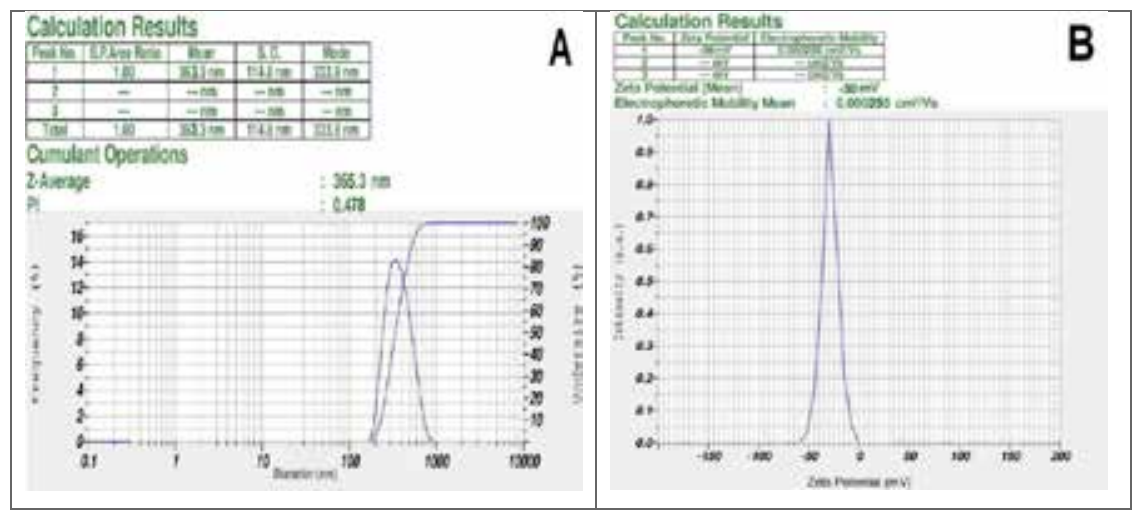

Figure 3: Particle size (A) and zeta potential (B) of optimized formulation

A stable vesicular system was predicted from the study of surface charge ${ }^{27}$. Therefore, the proposed design was capable to produce CFS loaded stable nano sized niosomes.

\section{Atomic force microscopy (AFM)}

The surface morphology of the niosomes were shown in figure 4, which revealed the formation of spherical and smooth surface niosomes. The AFM images in different scales further revealed the formation of spherical nano sized particles of similar size range as predicted by the design. 


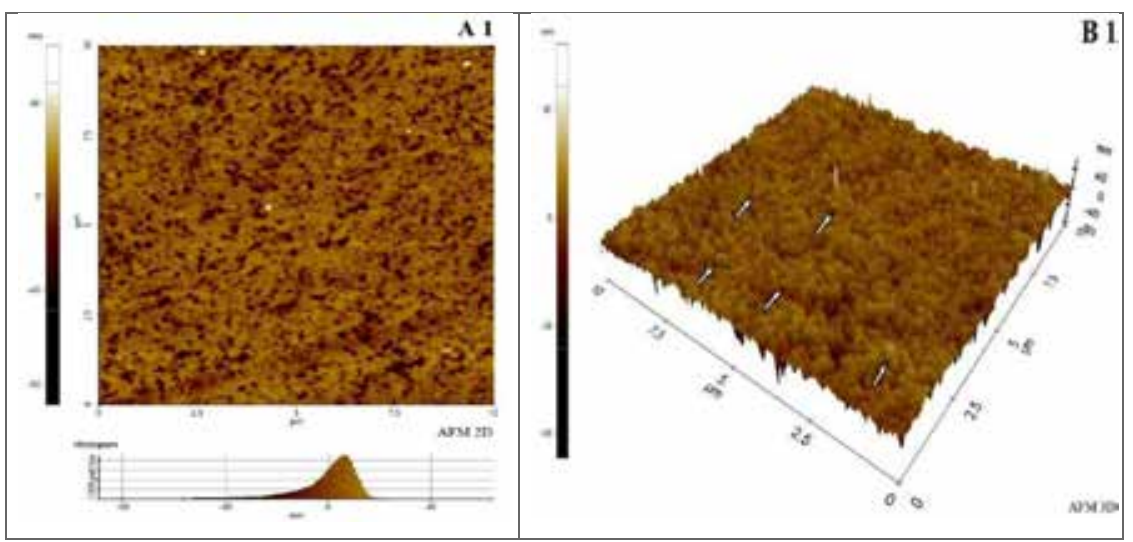

Figure 4: AFM images of optimized drug-loaded niosomes formulation (A1, 2D image B1, 3D Image)

\section{Differential scanning colorimetry (DSC)}

The DSC thermogram (figure 5) of the pure drug showed an endothermic peak corresponds to its melting point $180^{\circ} \mathrm{C}$, whereas the freeze-dried formulation (LYP-CFS-NIO) revealed a shift in the peak to $149{ }^{\circ} \mathrm{C}$, showing a decrease of phase transition temperature and heat $(\Delta \mathrm{H})$, which was an indication of the localization of drug inside the bilayer of lipids and surfactant. This agrees with the reported studies that the presence of cholesterol affects the gel liquid transition temperature of the vesicles ${ }^{28}$. Presence of long alkyl chain and hydrophilic moiety in tweens showed greater entrapment efficiency in niosomes while presence of cholesterol ensures greater bilayer stability ${ }^{29}$.

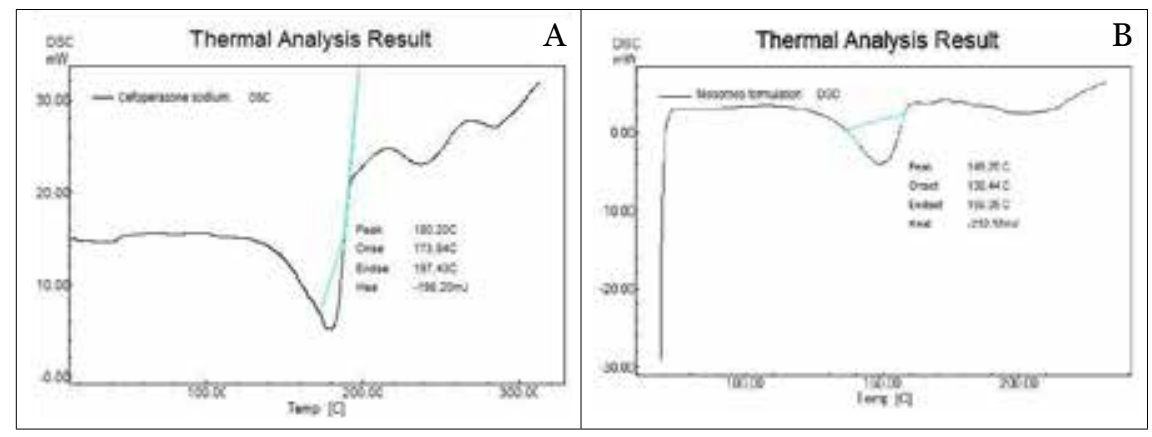

Figure 5: DSC thermograms of a pure $\operatorname{drug}(\mathrm{A})$ and Formulation (B). 


\section{X-ray powder diffraction (XRPD)}

The peak intensities of pure drug and the optimized freeze-dried formulations at various diffraction angles are represented in figure 6 . The pure drug showed high intensity peaks whereas the peak intensities of the same peak were reduced in the formulation graph. The high-intensity peaks of the pure drug represented its crystalline nature, while the optimized formulation showed the appearance of same peaks with low intensities. The low intensities of the peak area were attributed to the localization of the drug in the lipid and surfactant matrix and was an indication of high entrapment Which was in confirmation with DSC thermograms ${ }^{16}$.
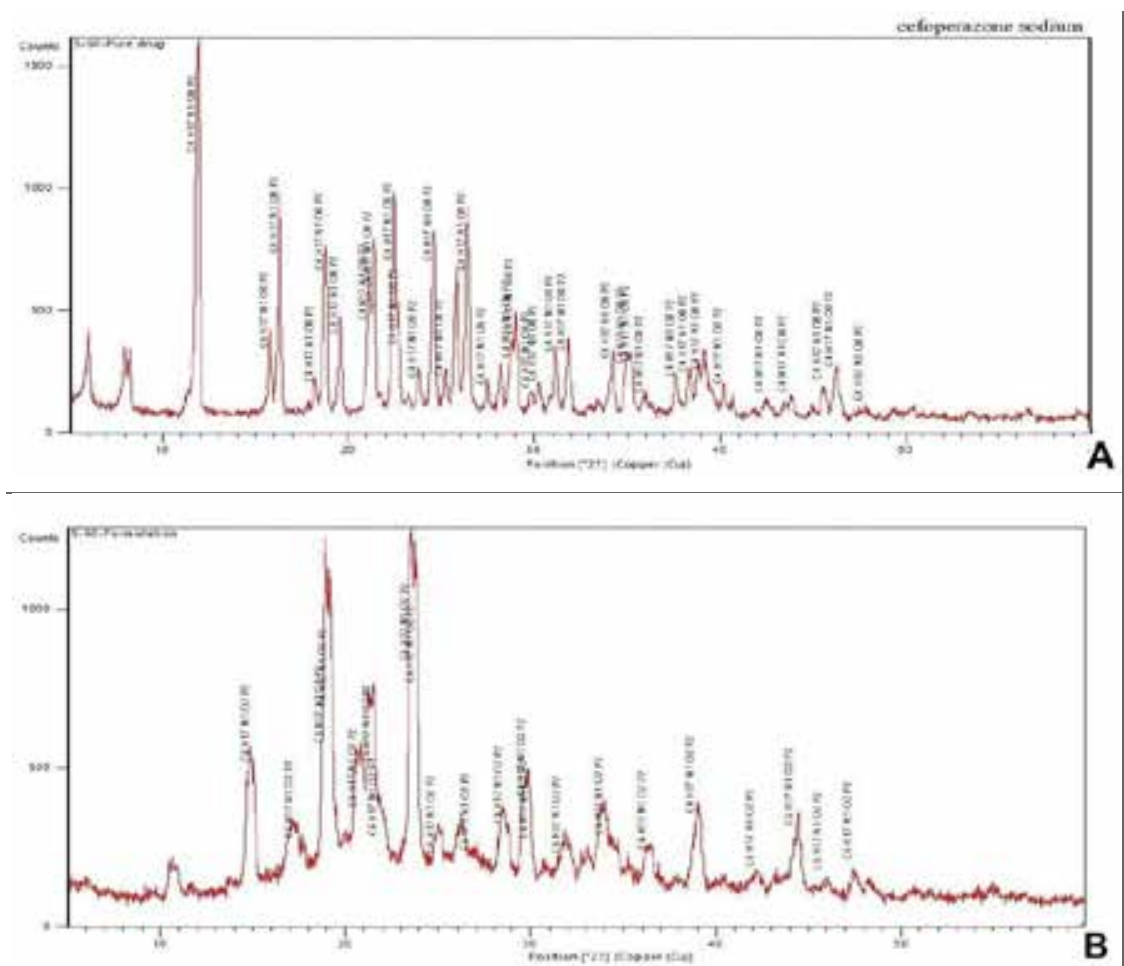

Figure 6: PXRD patterns of a pure drug $(A)$ and formulation $(B)$ 


\section{Evaluation of gel}

The LYP-CFS-NIO-gel showed a pH around 5.5 optimal to the skin conditions, a viscosity, drug content and spreadability of $84.13 \pm 0.25 \mathrm{cps}, 95 \pm 0.52 \%$, and $7.63 \pm 0.125 \mathrm{gm} . \mathrm{cm} / \mathrm{sec}$ respectively. Therefore, the gel was found to be suitable for dermal application based on its $\mathrm{pH}$, mechanical and rheological properties.

\section{In-vitro release and kinetics study}

The in-vitro release of the LYP-CFS-NIO-gel revealed a slow release of drug upto $86 \%$ in 8 hours as presented in figure 7. The release kinetics followed Higuchi model as per the highest regression coefficient value $\left(\mathrm{R}^{2}\right)$. The KorsmeyerPeppas modelling yielded a release component " $n$ " value of 0.57 , indicated that the drug release followed quasi-Fickian diffusion model with a matrix swelling and diffusion of the drug from the formulation.

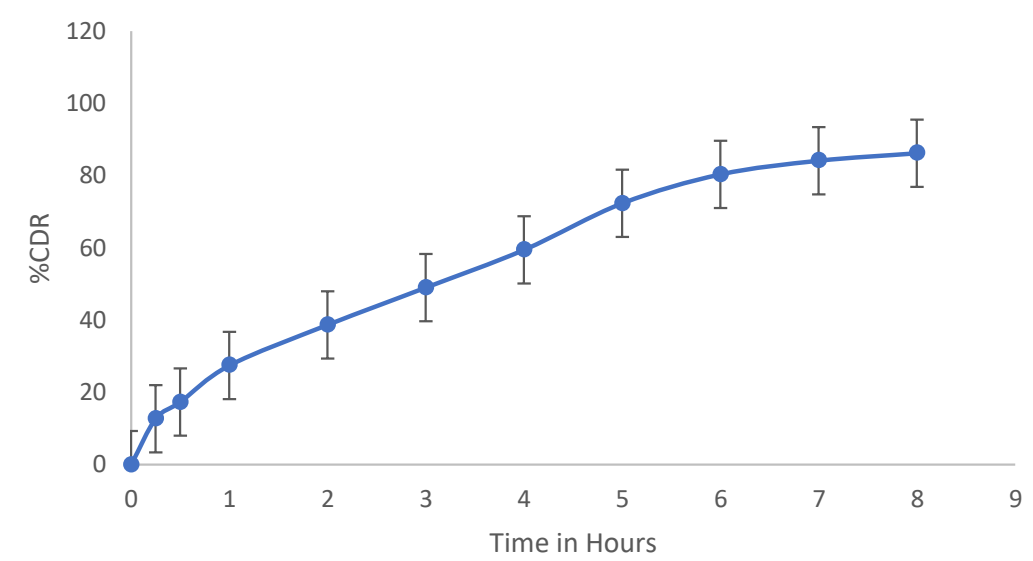

Figure 7: In-vitro release study of niosomal gel of CFS.

\section{Ex-vivo permeation study}

The ex-vivo permeation study unveiled that the niosomal gel showed higher penetration than the non niosomal gel of pure drug as shown in figure 8. It was further proved by the calculation of permeation parameters as mentioned in table 4 . The niosomal gel had a 3.28 times higher flux value than the non-niosomal gel of cefoperazone sodium. This observations attributes to the formulation characteristics, as niosomes can be effectively used as permeation enhancer. 


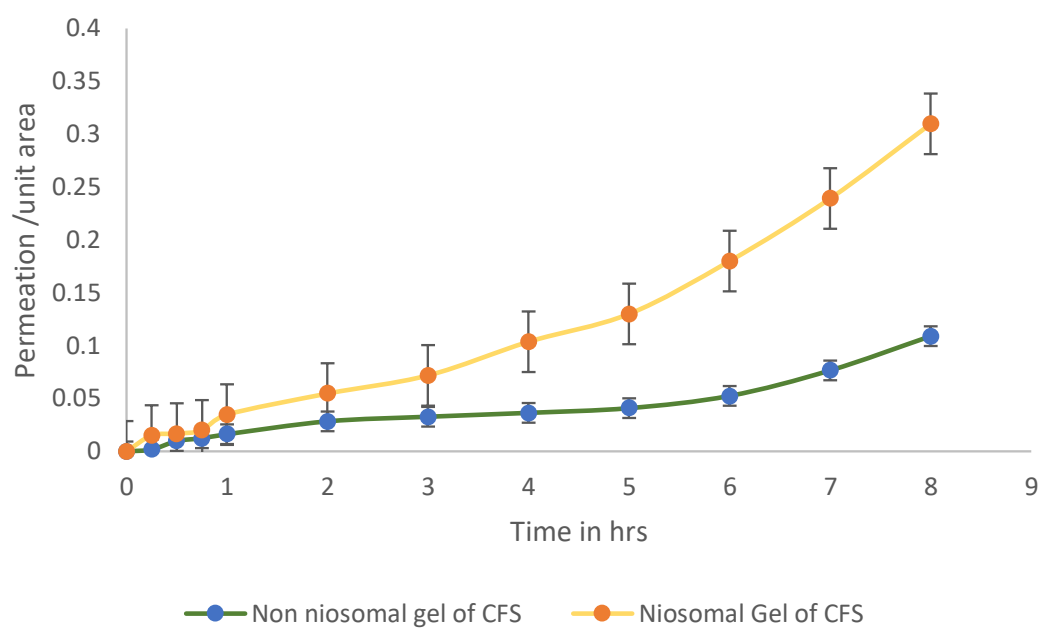

Figure 8: Ex-vivo permeation study of niosomal gel and non niosomal gel of CFS.

Table 4: Permeation parameters

\begin{tabular}{|c|c|c|c|}
\hline Formulation & $\begin{array}{c}\text { Steady state flux(mg/ } \\
\left.\mathbf{c m}^{2} / \mathbf{h r}\right)\end{array}$ & $\begin{array}{c}\text { Permeation constant (Kp) } \\
\mathbf{c m} / \mathbf{h r}\end{array}$ & $\begin{array}{c}\text { Drug permeation at 8 } \\
\text { hours (mg/cm }\end{array}$ \\
\hline LYP-CFS-NIO-gel & 0.032 & 0.016 & 0.309 \\
\hline Pure drug gel & 0.009 & 0.005 & 0.108 \\
\hline
\end{tabular}

\section{Skin irritation study}

The results of the skin irritation study revealed that following three days application of CFS-NIO- gel, there was no signs of skin irritation, no evidence on patchy or severe erythema associated with edema. Therefore, the gel was found to be suitable for dermal application.

The present study was designed to develop a novel delivery of cefoperazone sodium in niosomal gel for the treatment of skin infections. A highly permeable gel was prepared with niosomal cefoperazone sodium in Carbopol base, by screening of factors with custom design using JMP V13 software. The CFSNIO were characterized by their particles size, drug entrapment efficiency and surface charge. The optimized formulation was evaluated for AFM, DSC, XRPD studies and revealed the formation of nanosized stable vesicles with high encapsulation of drug. The CFS-NIO loaded Carbopol 934 gel showed its good mechanical and rheological property. The in-vitro release study showed a quasi Fickian release of the drug. The ex-vivo permeation and skin irritation study proved the improved penetrability and suitability of the gel for dermal appli- 
cation, respectively. Therefore, an effective transdermal delivery of CFS can be made with the prepared niosomal gel of CFS. These findings can create a paradigm for future studies for superior delivery of cefoperazone sodium in the treatment of skin infections.

\section{ACKNOWLEDGEMENT}

We are grateful for the encouragement and support of the management and principal of Krupanidhi College of Pharmacy, Bangalore to conduct the investigation. We extend our gratitude to IISC Bangalore for their support. 


\section{REFERENCES}

1. Moghassemi S, Hadjizadeh A. Nano-niosomes as nanoscale drug delivery systems: An illustrated review. J Control Release. 2014;185: 22-36.

2. Mahale NB, Thakkar PD, Mali RG, Walunj DR, Chaudhari SR. Niosomes: novel sustained release nonionic stable vesicular systems - an overview. Adv Colloid Interface sci. 2012; 183:46-54.

3. Jiao J. Polyoxyethylated nonionic surfactants and their applications in topical ocular drug delivery. Adv Drug Deliv Rev. 20o8; 60:1663-73.

4. Fathalla D, Abdel-Mageed A, Abdel-Hamid F, Ahmed M. In-vitro and in-vivo evaluation of niosomal gel containing aceclofenac for sustained drug delivery. Int J Pharm Sci Res. 2014; 1:105.

5. https://www.glowm.com/resources/glowm/cd/pages/drugs/co28.html.

6. https://www.drugbank.ca/drugs/DBo1329.

7. Liu T, Guo R, Hua W, Qui J. Structure behaviors of hemoglobin in PEG60oo/Tween 80/Span 80/H2O niosome system. Colloid Surf A Physicochem Eng Aspects. 2007; 293: 255-61.

8. Ibrahim MM, Shehata TM. The enhancement of transdermal permeability of water soluble drug by niosome-emulgel combination. J Drug Deliv Sci Technol. 2012;22(4):353-59.

9. Di Marzio L, Esposito S, Rinaldi F, Marianecci C, Carafa M. Polysorbate 20 vesicles as oral delivery system: In vitro characterization. Colloids and Surfaces B: Biointerfaces. 2013;104:20006.

10. Nahar S, Islam S, Khandaker S, Nasreen W, Hoque O, Dewan I. Formulation and Evaluation of Metoprolol Tartrate Loaded Niosomes Using 23 Factorial Design. J Pharm Res Int. 2018;22(6):1-17.

11. Srinivas S, Kumar YA, Hemanth A, Anitha M. Preparation and evaluation of niosomes containing aceclofenac. Dig J Nanomater Bios. 2010;5(1):249-54.

12. Bhattacharyya S, Sogali B. Application of Statistical Design to Assess the Critical Process Parameters of Ethanol Injection Method for the Preparation of Liposomes. Dhaka Univ J Pharm Sci. 2019;18(1):103-11.

13. Kumbhar D, Wavikar P, Vavia P. Niosomal Gel of Lornoxicam for Topical Delivery: In vitro Assessment and Pharmacodynamic Activity. AAPS Pharm Sci Tech. 2013;14(3):1072-82.

14. Gogoi H, Mani R, Bhatnagar R. A niosome formulation modulates the Th1/Th2 bias immune response in mice and also provides protection against anthrax spore challenge. Int $J$ Nanomed. 2018;13:7427-40.

15. Jin Y, Wen J, Garg S, Liu D, Zhou Y, Teng L, Zhang W. Development of a novel niosomal system for oral delivery of Ginkgo biloba extract. Int J Nanomed. 2013;8:421-30.

16. El-Sayed MM, Hussein AK, Sarhan HA, Mansour HF. Flurbiprofen-loaded niosomes-in-gel system improves the ocular bioavailability of flurbiprofen in the aqueous humor. Drug dev ind pharm. 2017;43(6):902-10.

17. Shirsand S, Kanani K, Keerthy D, Nagendrakumar D, Para M. Formulation and evaluation of Ketoconazole niosomal gel drug delivery system. Int J Pharm Investig. 2012;2(4):201-07.

18. Shilakari Asthana G, Asthana A, Singh D, Sharma P. Etodolac Containing Topical Niosomal Gel: Formulation Development and Evaluation. J Drug Deliv. 2016;2016:1-8.

19. Acharya A, Dhakal P and Khadka D: Formulation and Evaluation of Transdermal Gel of Lornoxicam and its Delivery by Passive and Inotophoresis Method: A Comparative Study. Int J Pharm Sci Res 2016; 7(2):810-18 
20. Narayana RC, Harish NM, Gulzar A M, Prabhakara P, Singh AK, Subrahmanyam EV. Formulation and in vitro evaluation of in situ gels containing secnidazole for vaginitis. Yakugaku Zasshi. 2009;129(5):569-74.

21. Singh S, Parashar P, Kanoujia J, Singh I, Saha S, Saraf SA. Transdermal potential and antigout efficacy of Febuxostat from niosomal gel. J drug deliv sci technol. 2017; 39:348-61.

22. Hardia A, Jamindar D, Mahajan A, Hardia A. Formulation and In Vitro and Skin Permeability Evaluation of Dexamethasone Loaded Niosomal Gel. Asian J Pharm Res Dev. 2017;5(2):1-9.

23. Payyal SP, Rompicherla NC, Sathyanarayana SD, Shriram RG, Vadakkepushpakath AN. Microemulsion Based Gel of Sulconazole Nitrate for Topical Application. Turk J Pharm Sci. 2020;17(3):259-64.

24. Arafa MG, Ayoub BM. DOE Optimization of Nano-based Carrier of Pregabalin as Hydrogel: New Therapeutic \&Chemometric Approaches for Controlled Drug Delivery Systems. Sci Rep. 2017; 7:41503.

25. Shirsand SB, Kumar GR, Keshavshetti GG, Bushetti SS, Swamy PV. Formulation and evaluation of cotrimoxazole niosomal gel for topical application. RGUHS. J Pharm Sci. 2015;5(1): 32-8.

26. Patel D, Dasgupta S, Dey S, Roja Ramani Y, Ray S, Mazumder B. Nanostructured lipid carriers (NLC)-based gel for the topical delivery of aceclofenac, preparation, characterization, and in vivo evaluation. Sci Pharm. 2012;80(3):749-64.

27. Detroja C, Chavhan S, Sawant K. Enhanced antihypertensive activity of candesartan cilexetil nanosuspension: formulation, characterization and pharmacodynamic study. Sci Pharm. 2011;79(3):635-51.

28. Kumar GP, Rajeshwarrao P. Nonionic surfactant vesicular systems for effective drug delivery-an overview. Acta Pharm Sin B. 2011;1(4):208-19.

29. Yeo LK, Olusanya TOB, Chaw CS, Elkordy AA. Brief Effect of a Small Hydrophobic Drug (Cinnarizine) on the Physicochemical Characterisation of Niosomes Produced by Thin-Film Hydration and Microfluidic Methods. Pharmaceutics. 2018;10(4):185. 